\title{
Optimization of Conditions for Cultivation of Babesia bigemina -infected RBCs
}

\author{
U. Rauf, I. Rashid, H. Akbar, K. Majeed ${ }^{1}$, I. Raheem, J. Nawaz, A. Durrani²
}

10.18805/IJAR.B-1276

\begin{abstract}
Background: Babesia bigemina is the cause of bovine babesiosis. B. bigemina-infected red blood cells (iRBCs) were passaged in vitro for the attenuation.

Methods: We cultured Pakistani isolate of the parasite in three different culture media. Whole blood was collected from splenectomized and intact crossbred young calves. The parameters included were hematological profile, catalase activity, osmotic fragility and lipid profile. Cell culture media (M-199, RPMI 1640 and DMEM) were used to find out the longevity of parasites iRBCs.

Result: Highest PPE level was found up to $6.0 \%$ on $72 \mathrm{~h}$ post-culture in M-199 medium. Furthermore, no significant difference in catalase activity while significant difference in osmotic fragility were observed. However, lipid profile was significantly less in infected animals except in Babesia-infected. M-199 was the most appropriate medium for the in vitro cultivation of B. bigemina. Our findings would help us for in vitro cultivation of babesia-infected RBCs for its attenuation.
\end{abstract}

Key words: Babesia bigemina, Culture medium, In vitro, Optimization, Percentage parasitized erythrocytes.

\section{INTRODUCTION}

Babesiosis is an economically important protozoan tick-borne disease of ruminants, which affects millions of animals around the globe (Terkawi et al., 2012) including Pakistan. Babesia bigemina ( $B$. bigemina) is highly prevalent in Pakistan and poses serious health and management issues to cattle (Jabbar et al., 2015). The most economical and effective method is vaccination for the control of babesiosis which improved the health and production of the animals (Babiuk, 2002). Moreover, implementation of mass vaccination program reduces the use of medicine and prevents the emergence of resistance for pathogens in animals (Lathers, 2001).

In vitro culture of infected cells is one of the most important techniques for the development of attenuated vaccine. The most important component for cell culture is selection of a suitable growth medium for in vitro cultivation of Babesiainfected cells. A culture medium in the presence of fetal bovine serum provides essential nutrients for proliferation of parasites (Arora, 2013; Morgan et al., 1955).

Erythrocyte's membrane is composed of polyunsaturated fatty acids that is vulnerable to lipid peroxidation (Esmaeilnejad et al., 2012), causing changes in levels of blood metabolites (iron, calcium and copper) and nutrients (proteins, antioxidant vitamins (,$C$ and $E$ ) and lipids) (Khan et al., 2011). Thus, babesia alters the hemato-biochemical profile including complete blood count (CBC), catalase activity and lipid profile and causes anemia during infection. The presence of $B$. bigemina in the erythrocytes of infected animals has hemolytic effect through increasing lipid peroxidation. In severe cases, there are possibilities of organ failure which leads to morbidity and mortality of infected animals (Turunç and Kontaş Aşkar, 2012).

The current study was designed to optimize the suitableculture medium for in vitro culture of Babesia-
Department of Parasitology, University of Veterinary and Animal Sciences, Lahore-54200, Punjab, Pakistan.

'Department of Physiology, University of Veterinary and Animal Sciences, Lahore 54200, Punjab, Pakistan.

${ }^{2}$ Department of Clinical Medicine and Surgery, University of Veterinary and Animal Sciences, Lahore 54200, Punjab, Pakistan.

Corresponding Author: I. Rashid, Department of Parasitology, University of Veterinary and Animal Sciences, Lahore-54200, Punjab, Pakistan. Email: Imran.rashid@uvas.edu.pk

How to cite this article: Rauf, U., Rashid, I., Akbar, H., Majeed, K., Raheem, I., Nawaz, J. and Durrani, A. (2021). Optimization of Conditions for Cultivation of Babesia bigemina -infected RBCs. Indian Journal of Animal Research. (): DOI: 10.18805/IJAR.B-1276.

Submitted: 09-04-2020 Accepted: 01-10-2020 Online: 11-02-2021

infected red blood cells (RBCs) as well as to evaluate the catalase activity, osmotic fragility and lipid profile of cattle infected with $B$. bigemina and/or Theileria annulata.

\section{MATERIALS AND METHOdS}

\section{Ethical statement}

The current study was approved from the animal welfare and ethic society of University of Veterinary and Animal Sciences Lahore, Pakistan with No. DR 1112, Dated: 1310-2017.

\section{Selection of animals}

A total of twelve piroplasm-free calves were undertaken in this study. The male crossbred calves of 4 to 6 months age were purchased from a local farm and were kept in experimental station of University of Veterinary and Animal Sciences Lahore, Pakistan. The animals were infected individually and collectively with ticks carrying $B$. bigemina 
Optimization of Conditions for Cultivation of Babesia bigemina -infected RBCs

and $T$. annulata reared at temperature $28^{\circ} \mathrm{C}$ and at humidity $>80 \%$ in Biological Oxygen Demand (BOD) incubator (Model ICO105 Memmert, Germany). The classification of the animals was done after screening of piroplasm infection in blood through PCR analysis. These calves were divided into four groups with each group carrying 3 animals. Group A consists of 3 crossbred splenectomized calf (Kusminsky et al., 1995 ) infected with ticks (containing $B$. bigemina) to use as a control positive, group $B$ includes calves infected with ticks (containing B. bigemina), group $\mathrm{C}$ consists of calves infected with ticks containing $B$. bigemina and $T$. annulata (mix infection), while group $D$ consists of healthy animals free from any infection to use as control negative. All reared calves were in free access to water and corn silage.

\section{Source of infection and parasitemia level in infected calves}

Calves of group A, B and C were exposed to tick infestation. After 14 days pre-exposure with ticks, blood samples from experimental animals were collected in EDTA coated tubes. Thin blood smear of these samples were prepared to examine the PPE. To calculate the parasitemia level in infected calves and in cultured RBCs, three smears from each sample were prepared to count the average number of $B$. bigemina/T. annulata-infected RBCs according to previously used method (Maharana et al., 2019). The PPE was measured by thin blood smear. Parasitemia level was calculated according to the previously reported formula (Schetters et al., 1994).

\section{Blood profile}

Complete blood count (CBC) of samples from experimental animals was performed by using hematological analyzer (Abbott Cell Dyn 4000). The Hb concentration (gm/dl), PCV (\%), RBCs count, WBC's count and other erythrocytic indices ( $\mathrm{MCV}, \mathrm{MCH}, \mathrm{MCHC}$ ) were compared among the four experimental animal groups.

\section{Catalase activity}

Whole blood was collected from venipuncture of jugular vein of individual experimental animals (A, B, C and D groups) in EDTA-coated tubes. These samples were processed for identification of antioxidant status by measuring catalase activity by spectrophotometer at $374 \mathrm{~nm}$ absorbance. Linear differential equation was used to determine the catalase activity (Hadwan and Abed, 2016).

\section{Preparation of Medium 199, RPMI 1640 and DMEM}

Medium 199 (Cat. No. 11150059), RPMI 1640 (Cat. No. 11875093) and DMEM (Cat. No. 11965092) were purchased from Thermo Fisher Scientific (Vanta, Finland). In this study, we cultivated the infected and non-infected RBCs in three different complete culture media following incubation in $5 \%$ $\mathrm{CO}_{2}$ at $37^{\circ} \mathrm{C}$ as previously conducted (Adaszek and Winiarczyk, 2011; Erp et al., 1980; Vega et al., 1985). The complete culture media were prepared comprising of $60 \%$ (Medium 199, RPMI 1640 and DMEM) with supplementation of FBS $40 \%$, amphotericin B $50 \mu \mathrm{g} \mathrm{ml}^{-1}$, penicillin $100 \mathrm{IU}$, streptomycin $100 \mathrm{\mu g} \mathrm{ml}^{-1}$ and HEPES $15 \mathrm{mM}$.

\section{In vitro cultivation}

Upon confirmation of infection and infection-free calves, blood samples were collected for in vitro propagation of $B$. bigemina in infected RBCs. A total of $10 \mathrm{ml}$ whole blood was collected from the calves of all 4 groups in $15 \mathrm{ml}$ EDTA coated tubes and thoroughly mixed. Live and dead cells were calculated by cell counting through hemocytometer. For that, trypan blue was used as a marker for the differentiation of live and dead cells (Rojas-Martinez et al., 2017). Centrifugation of collected blood samples was performed at $3000 \mathrm{rpm}$ for 6 minutes at $4^{\circ} \mathrm{C}$ to obtain the pellet of cells. The cells were washed 3 times with $0.15 \mathrm{M}$ phosphate buffer saline. The washed RBCs $(80 \mu \mathrm{l})$ were then cultured within each type of complete culture medium $\left(720 \mu\right.$ well $\left.^{-1}\right)$ in a 24-well culture plate and incubated in $5 \% \mathrm{CO}_{2}$ at $37^{\circ} \mathrm{C}$. After every $24 \mathrm{~h}, 700 \mu \mathrm{l}$ of culture media was replaced with fresh complete culture media. The infected RBCs were sub-cultured in vitro for 9 generations in duration of 36 days.

\section{Osmotic fragility of infected RBCs}

To perform erythrocytic osmotic fragility test, the most traditional protocol was followed (Parpart et al., 1947). Briefly, $\mathrm{NaCl}$ solution at percentage of $0.9,0.6,0.5,0.4,0.3$ and 0.0 in distilled water $\left(\mathrm{dH}_{2} \mathrm{O}\right)$ was used to optimize its concentration.

\section{Lipid profile activity}

The tests were performed for the profiling of three important parameters of lipids including cholesterol, triglycerides and high-density lipoprotein (HDL). Cholesterol analysis was done using Cholesterol LiquiColor® kit (Quiclet et al., 2019). Triglycerides analysis was done by using Kit (ab65336) (Zhang et al., 2015). HDL cholesterol level was measured by CHOLESTEROL liquicolor test kit (catalog no. INF $1001801 \mathrm{~GB})$ (Zhu et al., 2005).

\section{Statistical analysis}

RBCs of B. bigemina, T. annulata and in mix-infected calf were cultivated in vitro in three different media to find out the propagation of parasitemia. Their results were expressed in the form of percentage with respect to their media. Graphical analysis was done by using the GraphPad Prism 7 software (La Jolla, CA 92037, USA). One-way ANOVA was performed to analyze the catalase activity, osmotic fragility and lipid profile of the infected RBCs. The significance among the groups was evaluated by using Post Hoc test (Tukey's test). Significant values obtained were presented as ${ }^{*} p<0.05,{ }^{* *} p<0.01$ and ${ }^{* * *} p<0.001$ and "ns" represented non-significance $(p>0.05)$.

\section{RESULTS AND DISCUSSION \\ Parasitemia and Blood profile}

The results of parasitemia and blood profile are shown in Fig 1 and Fig 2, respectively.

We found decreased PCV (\%), Hb and RBC count in babesia-infected as compared to control negative group 
animals. All other hematological parameters were statistically non-significant among the groups. Our findings suggest that decrease in these parameters were due to destruction/lysis of infected RBCs. These results are concurrent by the previously reported changes in

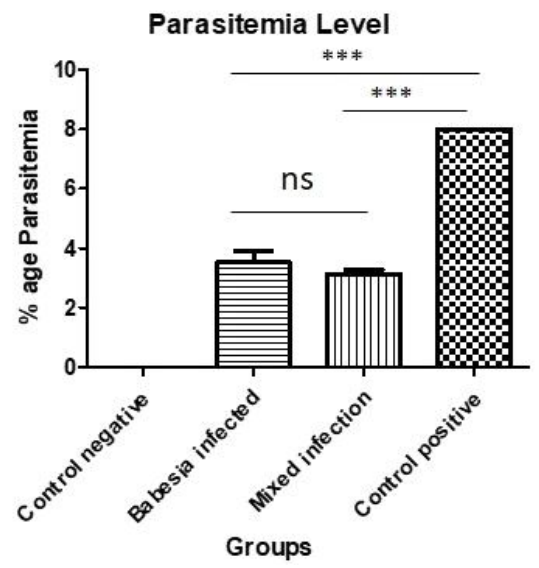

Fig 1: Parasitemia level (\%) in classified calves on 14 days posttick infestation.

Blood profile

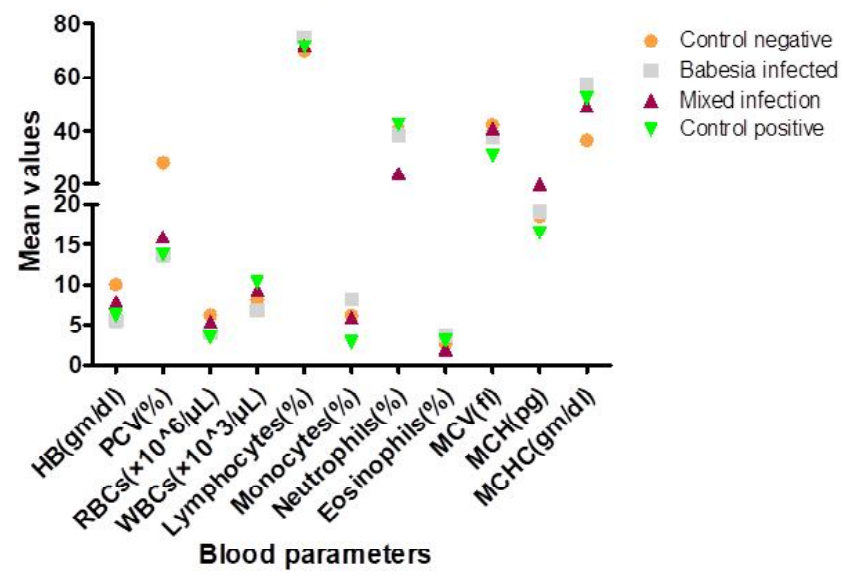

Fig 2: Complete blood profile (\%) of 4 groups calves at 14 day post-tick infection. hematology of $B$. bovis infected animals (Mahmoud and Abou-Zeina, 2008). In infected animals, WBC count was significantly $(<0.05)$ low as compared to control negative group while only neutrophil count was non-significant. High MCV and decreased MCHC were observed in control positive and babesia-infected animals respectively, but these values were non-significantly different. Decreased hematological values ( $\mathrm{MCHC}, \mathrm{RBCs}, \mathrm{PCV}$ and $\mathrm{Hb}$ ) suggested that anemia was the constant factor in babesiainfected animals. Our findings correlate with the previous study (Rahbari et al., 2008).

\section{Comparison of Media}

The results for the comparison of different media are shown in Table 1 and Fig 3. Out of culture media used, M-199 was found to be the most suitable for the proliferation of parasite in in vitro cultivation in RBCs and remained viable for duration of $>72 \mathrm{~h}$.

\section{Catalase activity}

The results of catalase activity are shown in Fig 4.

Non-significant difference in catalase activity among groups of calves infected with and without blood protozoan was found. Previous outcomes support our findings (Wang et al., 1999). According to the current findings, high level of catalase activity was observed in mix infection. Our findings are in close agreement to previous outcomes (Rezaei and Dalir-Naghadeh, 2006), where they found decreased catalase activity in healthy and severely anemic animals, while high catalase activity was recorded in mild to moderate Theileria-infected calves (Ahmed and Hassan, 2007; ElDeeb and Younis, 2009; Hamid et al., 2014; Razavi et al., 2012).

Table 1: Comparison of in vitro PPE in three different culture media.

\begin{tabular}{lcccc}
\hline \multirow{2}{*}{ Media } & \multicolumn{2}{c}{ PPE } & $\begin{array}{r}\text { Culture darkness } \\
\text { period (days) }\end{array}$ \\
\cline { 2 - 3 } & Initial & Final & 7 \\
Medium 199 & $1.58 \%$ & $6.0 \%$ & 3 \\
RPMI 1640 & $1.58 \%$ & $5.5 \%$ & 5 \\
DMEM & $1.58 \%$ & $5.75 \%$ & \\
\hline
\end{tabular}
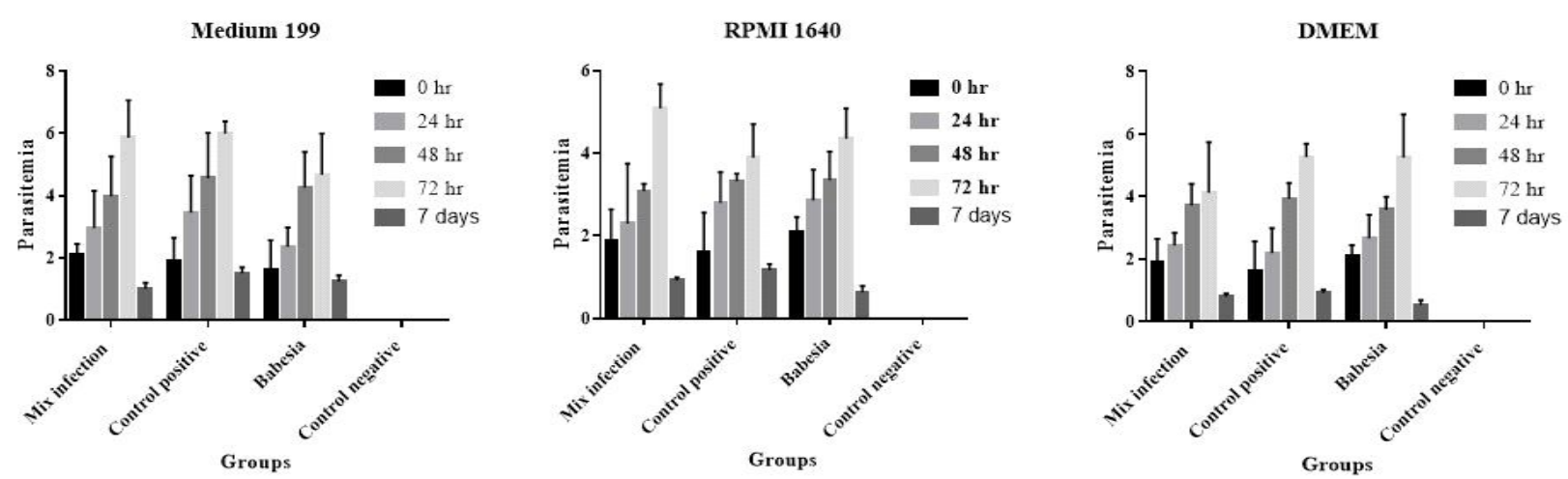

Fig 3: Percentage parasitemia in infected erythrocytes relative to culture medium and incubation time period. Parasitemia increased until $72 \mathrm{~h}$ while decreased beyond that incubation period due to addition of fresh RBCs. 


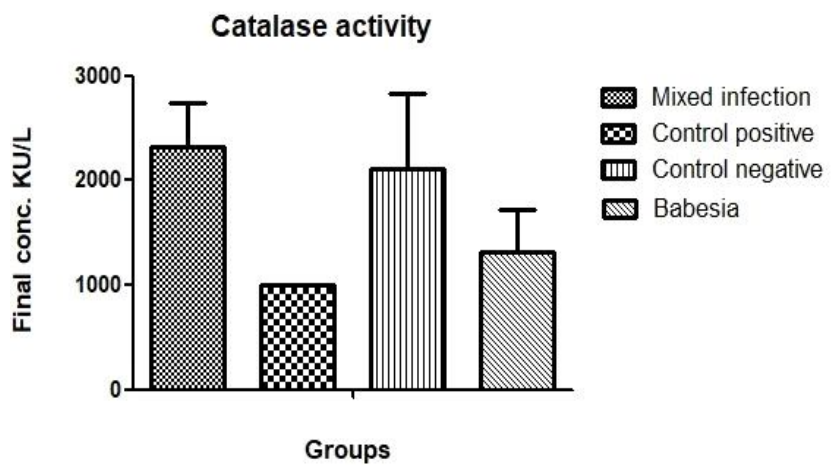

Fig 4: Graphical representation of catalase activity in 4 groups (A, $B, C$ and $D$ ) of calves. $X$-axis is showing the catalase activity in different groups and $\mathrm{Y}$-axis indicating the values of catalase activity in $\mathrm{KU} / \mathrm{L}$.

\section{Osmotic fragility}

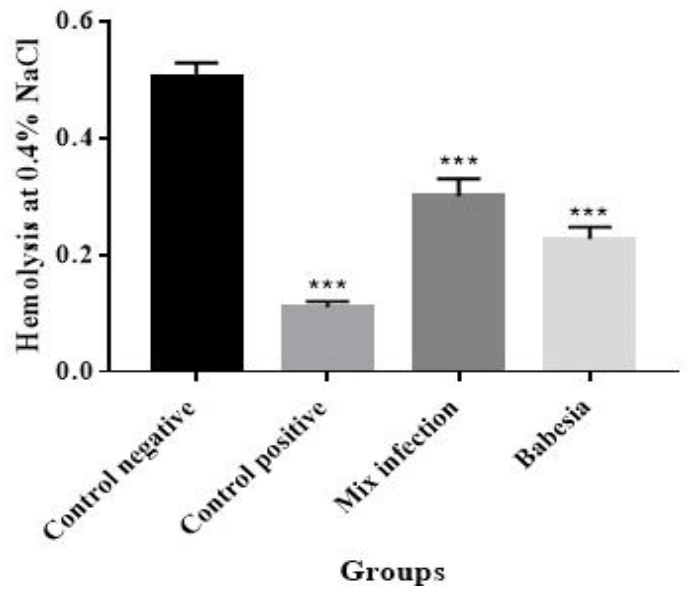

Fig 5: Graphical representation of erythrocytic osmotic fragility at $0.4 \% \mathrm{NaCl}$. X-axis showed the result of infected RBCs in classified animal groups while on $\mathrm{Y}$-axis represents hemolysis at $0.4 \% \mathrm{NaCl}$. There was no significant ( $p>0.05)$ difference between the animal groups.

\section{Osmotic fragility}

The results of osmotic fragility test are shown in Fig 5. Our results showed that erythrocytic osmotic fragility of mixed infected RBCs was statistically higher than of single infection. There might be several reasons including infection propagation, reduction of nutrient in culture media and hemolytic anemia (Fazio et al., 2016; Slappendel, 1998). Highest osmotic fragility was observed at the concentration of $0.4 \% \mathrm{NaCl}$ in all samples. The influx of water happens at $0.4 \% \mathrm{NaCl}$ because of hypo-tonicity of the culture media and the integrity of their membranes is disturbed. This disturbance permits the release of cell hemoglobin exterior to the cell membrane that dissolves in the external media. In mix-infection, there led to expand oxidative stress as shown by a significant rise in activities of chemicals like lipid peroxidation in erythrocytes. A noteworthy rise within the activities of antioxidant enzymes was observed which may not lower this oxidative stress. This could be the cause of increase in erythrocytic osmotic fragility because of membrane lysis and lower $\mathrm{Hb}$ values (Grewal et al., 2005). Noteworthy increase in erythrocytic osmotic fragility was in accordance with the previously reported studies in which the parasitemia expanded the erythrocytic osmotic fragility within Theileria sergenti-infected calves (Singh et al., 2001).

\section{Lipid analysis}

The results of lipid analysis are shown in Fig 6 .

Our findings were in contradiction with the results recorded by Goodger et al., (1981) in which the cholesterol and lipoproteins values were significantly decreased (Goodger et al., 1981). Cattle breed, susceptibility to infection and infection propagation could be the reasons for the difference in current and previous studies. Our findings for lipid profiles are supported with previous studies on cattle infected with babesiosis (Alam and Nasr, 2011). These results of triglycerides are similar with already findings that levels of triglycerides increased significantly (Turunç and Kontaş Aşkar, 2012). Similarly, we also found non-significantly increased levels of cholesterol and HDL in B. bigemina infected red blood cells (iRBCs) and these finding are
Cholesterol

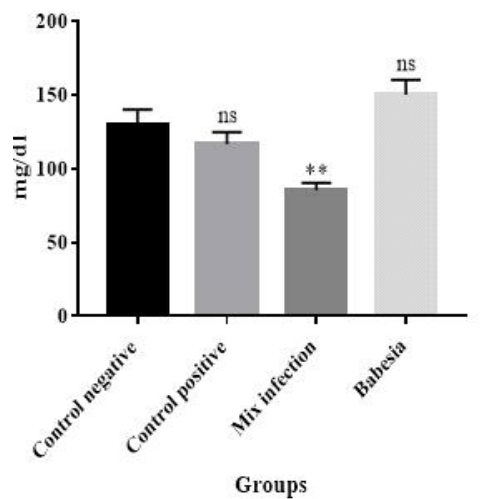

Triglycerides

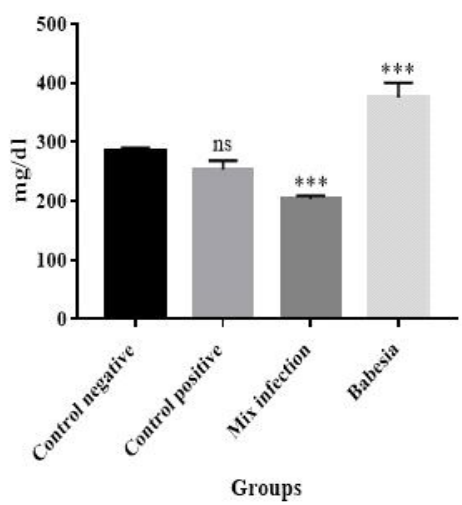

HDL

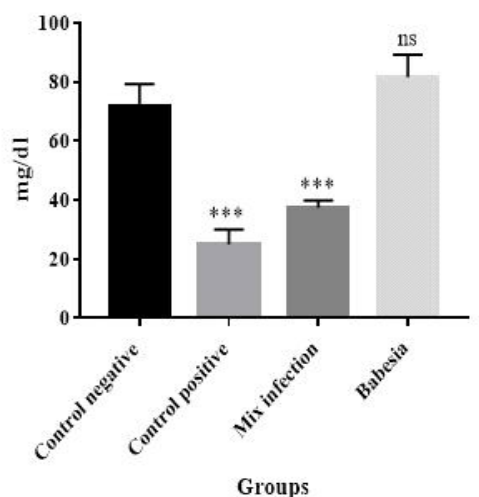

Fig 6: Graphical representation of lipid profile activity in in vitro cultured RBCs from classified animal groups. 
concurrent with previously reported study (Hamoda et al., 2014). Another study on cattle infected with B. bigemina showed a significant decrease $(P<0.05)$ in $H D L$ level and a non-significant decrease in the levels of other serum lipids (Ganguly et al., 2017). According to some previous studies, changes in lipid profiles were recorded non-significantly but our results noted only significant increase $(P<0.05)$ in the concentrations of triglycerides when compared with control group and the standards values (Razavi et al., 2012). The reason behind this deviation could be a change in cattle breed, susceptibility to infection and most importantly the parasitemia rate.

\section{CONCLUSION}

Non-significant changes in lipid profile showed that rate of $\mathrm{RBC}$ lysis was not higher in babesia-infected RBCs during in vitro cultivation. It is a satisfactory indicator to maintain $B$. bigemina in iRBCs for maximum duration in optimized culture medium to attenuate it for vaccine production against babesiosis in future studies.

\section{ACKNOWLEDGEMENT}

We are thankful to undergraduate Biological science female students; Iqra Tariq, Ramna Zia, Zauraise Mahnoor for performing the tests of this study during their last year internship program. Moreover, we are also thankful to Dr. Muhammad Rashid, Lanzhou Veterinary Research Institute, Chinese Academy of Agricultural Sciences, China for revising this manuscript.

\section{Funding}

Higher Education Commission, Pakistan (7173/Punjab/ NRPU/RandD/HEC/2017) has provided the grant for this study.

\section{Conflict of interest}

The authors declare that there is no conflict of interest.

\section{REFERENCES}

Adaszek, Ł. and Winiarczyk, S. (2011). In vitro cultivation of Babesia canis canis parasites isolated from dogs in Poland. Parasitol. Res. 108: 1303-1307.

Ahmed, W. and Hassan, S.E. (2007). Applied studies on coccidiosis in growing buffalo-calves with special reference to oxidant/ antioxidant status. World. J. Zool. 2: 40-48.

Alam, T. and Nasr, S. (2011). Hematological and biochemical investigation in bovine babesiosis and theileriosis. BVMJ. 22: 118-126.

Arora, M. (2013). Cell culture media: a review. Mater Methods. 3: 24. Babiuk, L. (2002). Vaccination: a management tool in veterinary medicine. Vet. J. 164: 188-201.

El-Deeb, W.M. and Younis, E.E. (2009). Clinical and biochemical studies on Theileria annulata in Egyptian buffaloes (Bubalus bubalis) with particular orientation to oxidative stress and ketosis relationship. Vet. Parasitol. 164: 301-305.

Erp, E., Smith, R., Ristic, M., Osorno, B. (1980). Continuous in vitro cultivation of Babesia bovis. American Journal of Veterinary Research. 41: 1141-1142.
Esmaeilnejad, B., Tavassoli, M., Asri-Rezaei, S. and Dalir-Naghadeh, B. (2012). Evaluation of antioxidant status and oxidative stress in sheep naturally infected with Babesia ovis. Vet. Parasitol. 185: 124-130.

Fazio, F., Casella, S., Giannetto, C., Giudice, E. and Piccione, G. (2016). Erythrocyte osmotic fragility in response to a short road transport in cattle, horses and goats. J. Vet. Behav. 12: 82-84.

Ganguly, A., Bisla, R., Ganguly, I., Singh, H., Bhanot, V. and Chaudhri, S. (2017). Direct blood PCR detection of Babesia bigemina and its effect on haematological and biochemical profile in crossbred cattle of eastern Haryana. Indian. J. Anim. Res. 51: 141-145.

Goodger, B., Wright, I. and Mahoney, D. (1981). Babesia bovis (Argentina): Studies of plasma lipids and lipoproteins during acute infections in cattle. Z. Parasitenkd. 65: 271276.

Grewal, A., Ahuja, C., Singha, S. and Chaudhary, K. (2005). Status of lipid peroxidation, some antioxidant enzymes and erythrocytic fragility of crossbred cattle naturally infected with Theileria annulata. Vet. Res. Commun. 29: 387394.

Hadwan, M.H. and Abed, H.N. (2016). Data supporting the spectro-photometric method for the estimation of catalase activity. Data Brief. 6: 194-199.

Hamid, O.M. A., Radwan, M.E. and Ali, A.F. (2014). Biochemical changes associated with babesiosis infested cattle. IOSR J. Appl. Chem. 7: 87-92.

Hamoda, A.F., Radwan, M., Rashed, R. and Amin, A. (2014). Toxic effect of babesiosis in cattle and chemotherapiotic treatment in Egypt. Am. J. Infect. Dis. Microbiol. 2: 91-96.

Jabbar, A., Abbas, T., Saddiqi, H.A., Qamar, M.F. and Gasser, R.B. (2015). Tick-borne diseases of bovines in Pakistan: major scope for future research and improved control. Parasites Vectors. 8: 283.

Khan, I.A., Khan, A., Hussain, A., Riaz, A. and Aziz, A. (2011). Hemato-biochemical alterations in cross bred cattle affected with bovine theileriosis in semi arid zone. Pak. Vet. J. 31: 137-140.

Kusminsky, R.E., Boland, J.P., Tiley, E.H. and Deluca, J.A. (1995). Hand-assisted laparoscopic splenectomy. Sur. Laparosc. Endosc. 5: 463-467.

Lathers, C.M. (2001). Role of veterinary medicine in public health: antibiotic use in food animals and humans and the effect on evolution of antibacterial resistance. J. Clin. Pharmacol. 41: 595-599.

Maharana, B.R., Kumar,B., Joseph, J.P. and Patbandha, T.K. (2019). A comparative analysis of microscopy and PCR based detection methods for Babesia and Trypanosoma infecting bovines and assessment of risk factors. Indian. J. Anim. Res. 53: 382-387.

Mahmoud, M.S. and Abou-Zeina, H.A. (2008). Current state in the serological diagnosis of babesiosis and haematological changes in splenectomised buffaloes. Glob. Vet. 2: 271281.

Morgan, J.F., Campbell, M. and Morton, H.J. (1955). The nutrition of animal tissues cultivated in vitro. 1. A survey of natural materials as supplements to synthetic medium 199. J. Natl. Cancer. Inst., 16: 557-567. 
Parpart, A.K., Gregg, J.R., Lorenz, P.B., Parpart, E.R. and Chase, A.M. (1947). Whole blood preservation; a problem in general physiology. An in vitro analysis of the problem of blood storage. J. Clin. Invest. 26: 641-654.

Quiclet, C., Dittberner, N., Gässler, A., Stadion, M., Gerst, F., Helms, A., Baumeier, C., Schulz, T.J. and Schürmann, A. (2019). Pancreatic adipocytes mediate hypersecretion of insulin in diabetes-susceptible mice. Metabolism. 97: 9-17.

Rahbari, S., Nabian, S., Khaki, Z., Alidadi, N. and Ashrafihelan, J. (2008). Clinical, haematologic and pathologic aspects of experimental ovine babesiosis in Iran. Iran. J. Vet. Res. 9: 59-64.

Razavi, S., Nazifi, S., Rakhshandehroo, E., Firoozi, P. and Farsandaj, M. (2012). Erythrocyte antioxidant systems, lipid peroxidation and circulating lipid profiles in cattle naturally infected with Theileria annulata. Rev. Med. Vet. 163: 18-24.

Rezaei, S.A. and Dalir-Naghadeh, B. (2006). Evaluation of antioxidant status and oxidative stress in cattle naturally infected with Theileria annulata. Vet. Parasitol. 142: 179-186.

Rojas-Martinez, C., Rodriguez-Vivas, R., Millán, J.F., Viana, K.A., Ruiz, E.G. and Martínez, J.Á. (2017). Putrescine: Essential factor for in vitro proliferation of Babesia bovis. Exp. Parasitol. 175: 79-84.

Schetters, T.P.M., Kleuskens, J.A.G.M., Scholtes, N.C., Pasman, J.W. and Bos, H.J. (1994). Vaccination of dogs against Babesia canis infection using antigens from culture supernatants with emphasis on clinical babesiosis. Vet. Parasitol. 52: 219-233.
Singh, A., Singh, J., Grewal, A. and Brar, R. (2001). Studies on some blood parameters of crossbred calves with experimental Theileria annulata infections. Vet. Res. Commun. 25: 289-300.

Slappendel, R. (1998). Abnormal osmotic fragility of erythrocytes in dogs and cats. Vet. Quart. 20: S38-S39.

Terkawi, M.A., Alhasan, H., Huyen, N.X., Sabagh, A., Awier, K., Cao, S., Goo, Y.K., Aboge, G., Yokoyama, N. and Nishikawa, Y. (2012). Molecular and serological prevalence of Babesia bovis and Babesia bigemina in cattle from central region of Syria. Vet. Parasitol. 187: 307-311.

Turunç, V. and Kontaş Aşkar, T. (2012). The determination of oxidative stress by paraoxonase activity, heat shock protein and lipid profile levels in cattle with theileriosis. Kafkas. Univ. Vet. Fak. Derg.18: 647-651.

Vega, C., Buening, G., Green, T. and Carson, C. (1985). In vitro cultivation of Babesia bigemina. Am. J. Vet. Res. 46: 416420.

Wang, Q., Murphy, N. and Black, S.J. (1999). Infection-associated decline of cape buffalo blood catalase augments serum trypanocidal activity. Infec. Immun. 67: 2797-2803.

Zhang, Y., Chen, M.I., Zhou, Y., Yi, L., Gao, Y.X., Ran, L., Chen, S.H., Zhang, T., Zhou, X. and Zou, D. (2015). Resveratrol improves hepatic steatosis by inducing autophagy through the cAMP signaling pathway. Mol. Nutr. Food. Res. 59: 1443-1457.

Zhu, W., Huang, X., He, J., Li, M. and Neubauer, H. (2005). Arterial intima-media thickening and endothelial dysfunction in obese Chinese children. Eur. J. Pediatr. 164: 337-344. 